\title{
La información como factor relevante en la actitud punitiva de los estudiantes
}

\author{
Deborah GARCÍA MAGNA \\ Universidad de Málaga \\ dgmagna@uma.es
}

Recibido: 08/10/2012

Aceptado: 23/01/2013

\begin{abstract}
Resumen
El presente artículo describe un proyecto docente y de investigación que pretende conseguir un doble objetivo: por un lado, profundizar en determinados contenidos de la materia que se imparte suministrando a los estudiantes información relevante sobre el sistema penal que no suelen obtener habitualmente en las clases y, por otro, investigar sobre la repercusión que tiene en la opinión de estos estudiantes el contar con dicha información adicional. En concreto, se pretende utilizar algunas técnicas docentes basadas en el uso de las TIC para identificar ciertas variables que pueden influir en las actitudes punitivas de los alumnos, como por ejemplo, contar con estadísticas sobre delincuencia y población penitenciaria, conocer y reflexionar sobre lo que opinan los expertos y analizar el tratamiento de la información que realizan los medios de comunicación.
\end{abstract}

Palabras clave: información, actitud punitiva, sistema penal, docencia e investigación

\section{Information as a Relevant Factor in the Punitive Attitude of Students}

\begin{abstract}
This paper describes a teaching and research project that aims to achieve two objectives: first, to deepen certain contents of the subject taught to students providing them with relevant information about the penal system that they normally don't get in the classroom; and second, to investigate on the impact that this information has on the opinion of students. Specifically, I intend to use some teaching techniques based on the use of ICT to identify certain variables that can influence punitive attitudes of students, for example, having statistics on crime and prison population, knowing and thinking about what experts say, or analizing the treatment of information carried out by the media.
\end{abstract}

Keywords: information, punitive attitude, penal system, teaching and research

Referencia normalizada

GARCÍA MAGNA, Deborah (2013): "La información como factor relevante en la actitud punitiva de los estudiantes". Estudios sobre el Mensaje Periodístico. Vol. 19, Núm. especial marzo, págs.: 207-212. Madrid, Servicio de Publicaciones de la Universidad Complutense.

Sumario: 1. Introducción; 1.1 Fuentes y estado de la cuestión; 1.2. Objetivos. 2. Metodología. 3. Desarrollo. 4. Conclusiones. 5. Referencias bibliográficas.

\section{Introducción}

\subsection{Fuentes y estado de la cuestión}

En un contexto como el actual, en el que todo puede ser objeto de controversia mediática, no es casual que en los últimos años se estén produciendo algunos procesos de discusión peculiares sobre temas que hasta entonces se habían mantenido alejados del debate público. Se ha convertido en algo natural el que se reiteren valoraciones extrajurídicas surgidas en torno a polémicos procesos legislativos y decisiones judiciales. En principio, este fenómeno no debería considerarse negativamente. Es esencial para la formación de la opinión pública en democracia que exista la posibilidad de discurrir libremente sobre lo que ocurre e intercambiar puntos de vista al respecto, algo que evidentemente no podría hacerse si no existiera un sistema de libre infor- 
mación. La situación, sin embargo, comienza a tornarse preocupante cuando algunas decisiones político-criminales se convierten básicamente en cuestiones de tinte electoralista y, como consecuencia, dependientes de una opinión pública no siempre bien informada y a la que previamente se le han proporcionado datos e informaciones descontextualizadas que provocan un estado de alarma y preocupación muchas veces infundado ${ }^{1}$. En ese sentido, el Derecho penal se ha convertido en objeto de discusión en la calle y en los medios, ante la mirada crítica de los expertos que alertan de que el uso excesivo de los instrumentos punitivos no es necesario ni eficaz. Sin embargo, cuando surgen conflictos en torno a cuestiones de tipo valorativo parece que la sociedad demanda o recibe de buen grado medidas jurídico-penales que, en un primer momento y precisamente por llevar aparejadas las sanciones más severas del ordenamiento jurídico, son consideradas como la expresión de una gran preocupación por los problemas sociales de parte de los poderes públicos, mientras que la adopción de políticas no penales es percibida en ocasiones como un signo de despreocupación. En efecto, las normas jurídico-penales constituyen la parte del Derecho que contiene las reglas básicas de convivencia y protege los valores más elementales, aplicando las sanciones más duras. Pero precisamente por ello debe usarse con moderación y sólo cuando sea imprescindible. Los ciudadanos en ocasiones basan sus actitudes ante el sistema penal y el delito de representaciones erróneas sobre la realidad de la delincuencia (creyendo que es más grave de lo que es) y sobre el sistema de justicia (considerando que es demasiado indulgente). La manera en que los poderes públicos gestionan estas situaciones (fomentándolas o suavizándolas) puede determinar que el sistema penal se convierta en una especie de baremo por el que se mida el estado de conformidad de la población con el programa político de cada momento, con el riesgo de restricción de libertades públicas y derechos que ello conlleva.

En definitiva, ante la realidad de unas sociedades mediatizadas, en las que los elementos de la organización política, económica y social se sitúan en el punto de mira de los medios de comunicación, la información se ha convertido en un bien vorazmente consumido por la población, siendo precisamente por ello uno de los medios más eficaces para conformar la opinión pública en un determinado sentido ${ }^{2}$.

\subsection{Objetivos}

El recurso desmedido a respuestas penales con el pretexto de aplacar a una opinión pública angustiada (a pesar de que las estadísticas demuestran que la delincuencia no es

\footnotetext{
1 Soto Navarro (2006) estudia cómo el tratamiento de la delincuencia en la prensa es una de las explicaciones del incremento de la preocupación por la inseguridad ciudadana, aunque no se corresponda con las cifras reales de delincuencia. Rechea Alberola y Fernández Molina (2006), también relacionan las preocupaciones de los españoles y el tratamiento de la información por los medios. McCombs $(2006,106)$, señala que "los ciudadanos están envueltos en un proceso de aprendizaje continuo sobre los asuntos públicos. Sus respuestas al cuestionario del encuestador sobre cuáles son los temas más importantes son un reflejo, por lo general, de las lecciones que imparten los medios en las cuatro u ocho semanas anteriores".

2 McCombs (1996) considera que los medios de comunicación son los actuales creadores de los estados de opinión, fijando la agenda sobre los temas a los que se les debe otorgar importancia y decidiendo cuáles no merecen cobertura mediática .
} 
un problema grave en España), la renuncia por parte de la sociedad a parcelas cada vez mayores de libertad para conseguir más seguridad en la vida cotidiana (cuando en realidad no existe una amenaza que justifique tal recorte de derechos) y el incremento de la presión punitiva en cada una de las fases y ámbitos del sistema penal son sólo algunos de los problemas que presentan los actuales procesos criminalizadores.

La docencia del Derecho penal en estas condiciones de partida resulta complicada. Los estudiantes, como el resto de los ciudadanos, viven en un contexto de exceso de información (a menudo inexacta o incompleta), y en consecuencia acusan una sensación de inseguridad creciente (aunque no provenga de la delincuencia) y desconocen la verdadera dimensión del delito en nuestro país (exagerando tanto su cantidad como su gravedad). En ocasiones los alumnos se muestran incrédulos ante la realidad de los datos que se les proporcionan o cuestionan la fiabilidad de las fuentes y la corrección de las estadísticas (sean o no oficiales), confrontándolas con su propia experiencia o la de terceras personas a quienes a veces ni siquiera conocen personalmente (sino a través de los medios de comunicación). Por otra parte, también es cierto que suelen ser receptivos a los argumentos que apoyan el respeto a las garantías, la firmeza de los principios en que se basan las normas y la necesidad de establecer unas bases claras que proporcionen seguridad jurídica.

Partiendo de esta realidad y siguiendo experiencias anteriores (García Magna y García España, 2011), pretendo llevar a cabo una investigación con la participación de los estudiantes que asisten a mis clases, utilizando para ello recursos que servirán al mismo tiempo como materiales de aprendizaje. Parto de la idea de que es necesario proporcionarles información sobre el sistema penal más allá de la que sería imprescindible para superar satisfactoriamente la asignatura. Además considero muy interesante analizar cómo les influye contar con esa información adicional, en tanto que ciudadanos con ideas preconcebidas sobre el sistema penal. En definitiva, aprovechando las ventajas del uso de las TIC en el ámbito docente, se pretende poner en práctica un proyecto de investigación diseñado para indagar en la influencia que el conocimiento sobre el sistema penal puede tener en la opinión de los estudiantes de Derecho y, en concreto, su repercusión sobre las actitudes punitivas.

\section{Metodología}

La investigación que se presenta combina una metodología cualitativa, basada en el análisis del discurso sobre temas concretos relacionados con la realidad de la política criminal actual a través de debates y diarios reflexivos, con el análisis cuantitativo de los datos obtenidos a partir de una encuesta de valoración y conocimiento sobre el sistema penal. Los participantes en el estudio son alumnos matriculados en la asignatura Derecho penal Parte general del Grado en Derecho de la Universidad de Málaga. Se trata de estudiantes que siguen el sistema de evaluación continua y, por tanto, asisten regularmente a las clases presenciales y realizan la mayoría de las actividades que se les proponen a lo largo del curso, tanto en el aula como en la plataforma virtual de apoyo a la asignatura. Entre todas las tareas propuestas, deben completar tres instrumentos de valoración: un cuestionario anónimo sobre actitudes punitivas con preguntas cerradas, un grupo de discusión y un diario reflexivo sobre tres situaciones 
delictivas distintas. Se pretende analizar su actitud punitiva de partida y comprobar si su opinión varía cuando se les proporciona más información sobre el sistema penal que la que obtienen habitualmente, analizando cómo valoran ellos ese eventual cambio de actitud ante la delincuencia y el sistema penal. Para estudiar el posible cambio producido, el primero de los instrumentos de trabajo es una encuesta anónima sobre actitudes punitivas, que se les debería pasar al principio del curso y de nuevo cuando acaben las clases. Se trata de una encuesta de elaboración propia, a partir de la diseñada por García Magna y García Leiva (2012). Se compone de 34 preguntas: 11 respecto al perfil del encuestado y variables que pueden influir en su actitud punitiva; 7 sobre el conocimiento que tienen del sistema penal español; y 13 sobre su opinión al respecto. Todas las preguntas son de respuesta única, excepto una que permite exponer ideas o detallar respuestas.

El segundo instrumento de investigación es el grupo de discusión. Se pretende con él que todos los alumnos que ya han realizado el cuestionario también lleven a cabo esta actividad como parte del sistema de evaluación continua de la asignatura. Así, los participantes en el foro asisten regularmente a clase y, por tanto, tienen niveles similares de conocimiento sobre el sistema penal en su conjunto. En el grupo de discusión se llevan a cabo debates acerca del anteproyecto de reforma del Código penal español consistente en incorporar la pena de prisión permanente revisable ${ }^{3}$.

Para terminar, el tercer instrumento de análisis supone la realización por el estudiante de un diario reflexivo en el que plasme su opinión personal acerca de tres supuestos de hecho que se le plantean y a los que se podría aplicar la pena de prisión permanente revisable. Las circunstancias de los casos prácticos se detallarán de forma que se proporcionen al estudiante datos tanto del sujeto que comete el delito como de las condiciones en que éste se ha producido. Para llevar a cabo esta actividad el estudiante ya debe contar con un bagaje suficiente sobre Derecho penal, por lo que se realizará a final de curso y previamente se pondrán a su alcance una serie de textos jurídicos y noticias relacionadas con el tema.

\section{Desarrollo}

Mediante este proyecto se pretenden aunar las actividades docente e investigadora a través del uso de las tecnologías de la información y la comunicación, intentando que los mismos materiales que sirven de instrumento para el análisis de la actitud punitiva de los estudiantes les proporcionen un enfoque amplio (Terradillos Basoco, 2008, 6) y duradero (Bain, 2005, 28), de carácter político-criminal y que no limite el aprendizaje a la memorización de conceptos jurídicos.

La información objeto de análisis se obtiene en tres fases: a comienzos de curso mediante el cuestionario anónimo (para estudiar la actitud y conocimiento de partida) y el foro de debate (opinión sobre la cadena perpetua); a mediados de curso, mediante

${ }^{3}$ En un principio esta sanción supondría un mínimo de entre 25 y 35 años de prisión antes poder acceder a cualquier posibilidad de revisión y sería aplicable a casos de terrorismo, magnicidios, genocidios y ciertos supuestos de asesinato agravado. 
el diario reflexivo sobre supuestos concretos a los que eventualmente se podría aplicar la pena de prisión permanente revisable; y al finalizar el curso mediante un nuevo pase del cuestionario anónimo (comparando los datos obtenidos con el pase previo).

Se parte de la hipótesis de que los estudiantes se mostrarán al principio moderadamente punitivos y que irán suavizando su actitud a medida que reciban información sobre el sistema penal y deban reflexionar al respecto. Para ello se les pedirá, entre otras cosas, que valoren si, con las cifras reales de delincuencia en la mano consideran necesario el endurecimiento de las penas (concretamente, la implantación de la cadena perpetua en nuestro ordenamiento). Si su respuesta es positiva, se les pedirá que reflexionen sobre cuál es el fin de la pena que la reforma estaría primando sobre los demás (si el inocuizador, el preventivo o el resocializador), debiendo analizar qué está sucediendo en la práctica, en base a una serie de premisas que ellos mismos descubrirán a lo largo del curso (ODA, 2011), como por ejemplo, el abuso de las penas de prisión en el ámbito legislativo y judicial; el hecho de que alrededor del $80 \%$ de los reclusos los sean por delitos contra el patrimonio y de menudeo de drogas (es decir, delincuencia callejera, que suele provenir de poblaciones marginales, normalmente por causas socioeconómicas); el que las cárceles estén masificadas a pesar de que nuestras cifras de delincuencia no son alta; o la dificultad que existe de llevar a cabo programas dirigidos a la reinserción social y, por tanto, a evitar la reincidencia.

\section{Conclusiones}

La experiencia de años anteriores en los que he aplicado varias partes y fases de este proyecto de manera aislada, junto a la literatura e investigaciones existentes sobre la materia, me permite poder adelantar algunas hipótesis que creo que se podrán ver confirmadas al finalizar el curso académico, referidas no sólo a la investigación sobre el posible cambio de actitud punitiva gracias a la información recibida, sino también a los resultados académicos obtenidos mediante esta metodología docente.

En una fase inicial, los estudiantes que deben dar su opinión sobre el sistema penal contando con la misma información que el resto de la población, suelen mostrar una actitud severa hacia el delincuente, en especial si identifican el tema con algún suceso concreto del que han tenido noticia a través de los medios de comunicación. Sin embargo, al reflexionar sobre un supuesto de hecho conociendo detalles del caso y de la persona que ha cometido el delito, suelen ser más indulgentes que los propios jueces y menos severos que algunas de las normas contenidas en los códigos penales, incluso si todavía carecen de información sobre la realidad de la delincuencia y el sistema penal.

Es curioso comprobar año tras año cómo conforme adquieren más conocimiento sobre el sistema penal en su conjunto, sus opiniones se van moderando. En definitiva, a medida que conocen cómo es y cómo debería ser el sistema penal, qué carencias tiene la regulación actual y qué críticas hacen los expertos, cómo son realmente las instituciones que aplican y ejecutan las normas (por ejemplo acudiendo a juicios o visitando un centro penitenciario), su actitud punitiva se suaviza.

Aunque algunos estudiantes no muestran una actitud abierta al aprendizaje activo en un principio, la realidad demuestra que cuando se les implica en este tipo de metodologías, suelen comprender que los conocimientos adquiridos son útiles y que mu- 
chas de las conclusiones a las que llegan son el fruto de un esfuerzo personal de trabajo y reflexión, lo que les motiva para seguir aprendiendo.

La investigación que se acaba de presentar en estas páginas sería casi imposible de completar sin la ayuda de las tecnologías de la información y la comunicación puestas al alcance del docente en las universidades actuales. Es indudable que las TIC facilitan enormemente la recogida de datos y la tarea de análisis del investigador, garantizando una comunicación fluida entre los estudiantes ( $\mathrm{y}$ con el profesor), $\mathrm{y}$ ofreciendo amplísimas posibilidades de hacerles llegar recursos y materiales, y de obtener retroalimentación acerca del proceso de aprendizaje activo en el que se encuentran.

\section{Referencias bibliográficas}

BAIN, Ken,(2005): Lo que hacen los mejores profesores universitarios. Valencia, Publicaciones de la Universidad de Valencia.

GARCÍA MAGNA, Deborah, y GARCÍA ESPAÑA, Elisa (2011): "La investigación criminológica como metodología docente innovadora", en Actas del VIII Congreso Español de Criminología. SEIC.

GARCÍA MAGNA, Deborah, y GARCÍA LEIVA, Patricia, (2012): "La percepción de los ciudadanos acerca de la prisión. Estudio sobre actitudes punitivas de estudiantes de Derecho ante la experiencia de la visita a un centro penitenciario", en Actas del IX Congreso Español de Criminología. SEIC.

MCCOMBS, Maxwell (1996): "Influencia de las noticias sobre nuestra imagen del mundo", en BRYANT, Jennings y ZILLMANN, Dolf (comps.): Los efectos de los medios de comunicación. Investigaciones y teorías. Barcelona, Editorial Paidós.

MCCOMBS, Maxwell (2006): Estableciendo la agenda: El impacto de los medios en la opinión pública y en el conocimiento. Barcelona, Editorial Paidós.

ODA, Observatorio de la delincuencia (2012): Realidad y política penitenciarias. Informe ODA 2010/2011. Instituto Andaluz Interuniversitario de Criminología, Sección de Málaga.

RECHEA ALBEROLA, Cristina, y FERNÁNDEZ MOLINA, Esther (2006): Los discursos sobre seguridad ciudadana y lucha contra la delincuencia en la prensa española. Centro de Investigación en Criminología, Universidad de Castilla La Mancha Informe $\mathrm{n}^{\mathrm{o}} 13$.

SOTO NAVARRO, Susana (2006): "Delincuencia en la prensa", en Boletín Criminológico, $\mathrm{n}^{\mathrm{o}}$ 83, enero-febrero, Instituto Andaluz Interuniversitario de Criminología.

TERRADILLOS BASOCO, Juan María (2008): "Sobre el sentido de la enseñanza del Derecho penal", en Revista de Derecho penal, nº 17.

\section{Deborah GARCÍA MAGNA}

Universidad de Málaga

Profesora de Derecho Penal

dgmagna@uma.es 